\title{
Intra-articular synovial sarcoma in the ankle joint: A case report
}

\author{
TOMOKI NAKAMURA, TOMOHITO HAGI, KUNIHIRO ASANUMA and AKIHIRO SUDO \\ Department of Orthopaedic Surgery, Mie University Graduate School of Medicine, Tsu, Mie 514-8507, Japan
}

Received February 16, 2018; Accepted July 26, 2018

DOI: $10.3892 / \mathrm{mco} .2018 .1702$

\begin{abstract}
Intra-articular primary soft tissue sarcomas are extremely rare. The present case report describes a case of intra-articular synovial sarcoma of the ankle joint. A 51-year-old man presented with complaints of swelling and progressive pain in the right ankle. Lateral radiographs of the ankle revealed lytic invasion of the anterior and posterior parts of the talus. Computed tomography imaging revealed a low-density lesion with calcifications around the ankle joint. Magnetic resonance imaging revealed that the diffuse lesion was isointense on T1-weighted images and hypointense to hyperintense on $\mathrm{T} 2$-weighted images. The final diagnosis was synovial sarcoma. The foot could not be salvaged as the sarcoma was diffusely spread throughout the ankle joint; thus, the patient underwent below-the-knee amputation with neoadjuvant and adjuvant chemotherapy. He succumbed from fatal arrhythmia 2 years following the initial diagnosis. Although intra-articular primary soft tissue sarcomas are extremely rare, the possibility of malignancy should be considered.
\end{abstract}

\section{Introduction}

Intra-articular primary soft tissue sarcomas are extremely rare. In the few cases that have been published, it mostly arose in the knee joint of adults, with the most common type being synovial sarcoma (1-3). Synovial sarcoma accounts for 5 to $10 \%$ of soft tissue sarcomas and typically occurs in patients between 15 and 35 years (4). Synovial sarcoma is unrelated to the synovium, and less than 5\% cases originate within a joint $(1,4)$, which mostly occurs in the knee joint (1-3). Yet, other locations have been reported, with three cases involving the elbow and one involving the hip joint (1). Furthermore, one case of intra-articular synovial sarcoma at the ankle joint was described in a previous report in which the magnetic resonance imaging (MRI) features of 12 synovial sarcomas were reviewed; however, this report provided no detailed clinical information (5). In this report, we describe a case of intra-articular synovial sarcoma of the right ankle

Correspondence to: Dr Tomoki Nakamura, Department of Orthopaedic Surgery, Mie University Graduate School of Medicine, 2-174 Edobashi, Tsu, Mie 514-8507, Japan

E-mail: tomoki66@clin.medic.mie-u.ac.jp

Key words: synovial sarcoma, intra-articular, ankle joint in a 51-year-old man. The requirement for institutional review board approval of our institute was waived owing to the anonymized and retrospective nature of this report, but we obtained written informed consent to perform future studies from the patient.

\section{Case report}

A 51-year-old man was referred to out hospital due to swelling and progressive pain in the right ankle that was present for 2 months. There was no history of trauma, and his medical history was otherwise unremarkable. Physical examination of his right ankle revealed a painful yet nearly full range of motion as well as tenderness, particularly on the anterior part of the ankle joint. Lateral radiographs of the ankle revealed lytic change of the anterior and posterior parts of the talus (Fig. 1). Computed tomography (CT) imaging revealed a low-density lesion with calcifications around the ankle joint (Fig. 2). MRI indicated that the diffuse lesion was isointense on T1-weighted images and hypointense to hyperintense on T2-weighted images. After administration of gadolinium-containing contrast medium, the tumor revealed heterogeneous enhancement (Fig. 3). Open biopsy was performed. Histological findings revealed a monophasic malignancy composed of spindle cells. Immunohistochemically, the tumor was positive for BCL2 and CD99/MIC2 and focally positive for epithelial membrane antigen and $\alpha$ smooth muscle actin, but negative for desmin, CD34, and cytokeratins. The aforementioned morphologic and immunohistochemical findings were consistent with monophasic synovial sarcoma. The foot could not be salvaged because the sarcoma was diffusely spread throughout the ankle joint; thus, the patient underwent below-knee amputation with neoadjuvant (three courses) and adjuvant (two courses) chemotherapy using gemcitabine and docetaxel. After rehabilitation, he used a below-the-knee prosthesis and walked without any need for support. One year after the initial treatment, lymph node metastasis at the popliteal vasculature and thigh developed. Therefore, above-knee amputation with adjuvant chemotherapy using adriamycin and ifosfamide was performed. However, multiple lung metastases developed after 3 months. He had started chemotherapy using eribulin, but he suddenly died from fatal arrhythmia, which were not related to eribulin, 2 years after the initial diagnosis.

\section{Discussion}

Synovial sarcoma accounts for 5 to $10 \%$ of all primary soft tissue sarcomas (4). Despite its name, whether synovial 

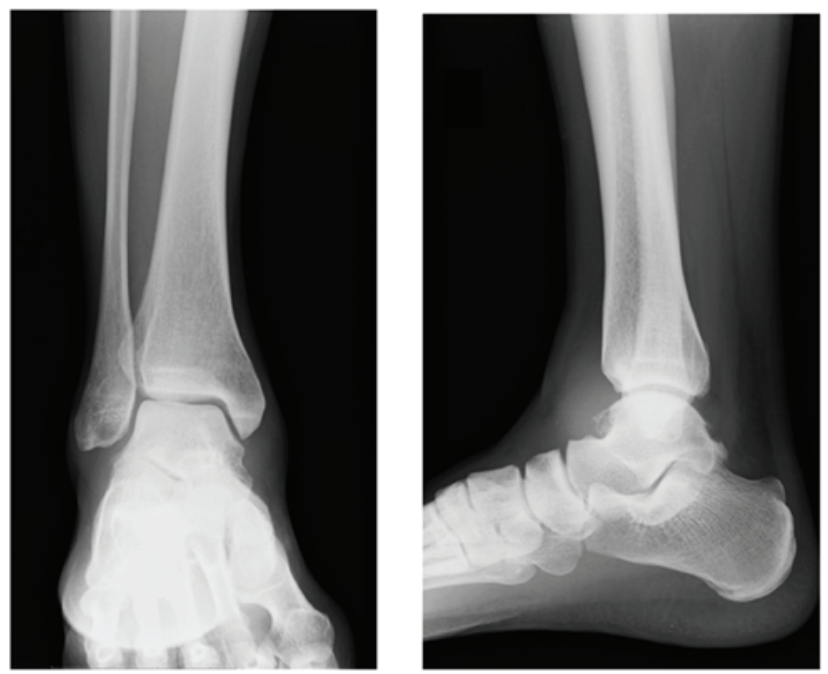

Figure 1. Lateral radiograph presenting lytic change of the anterior and posterior parts of the talus (right, A-P view; left, lateral view).
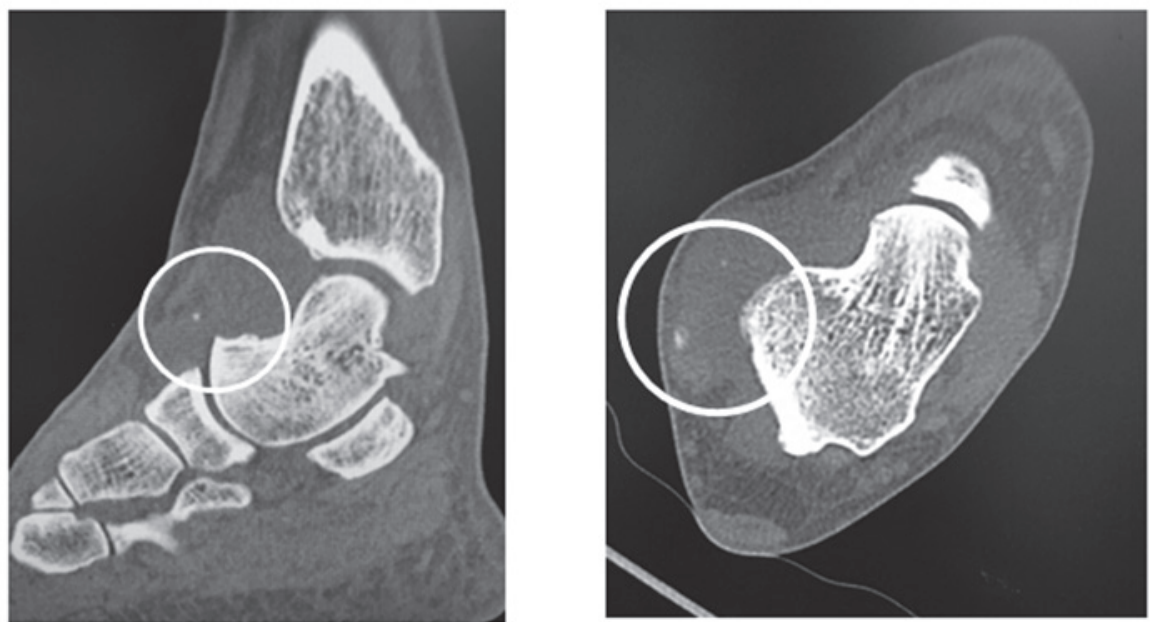

Figure 2. Computed tomography image revealing a low-density lesion with calcification (circle) around the ankle joint.

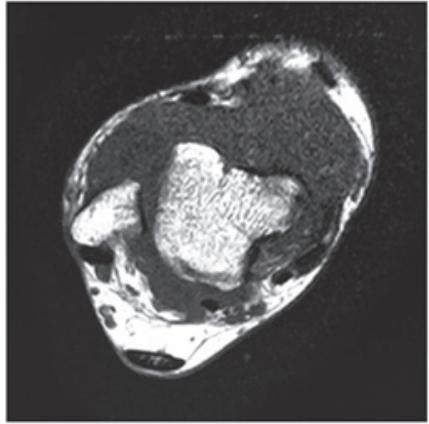

T1WI

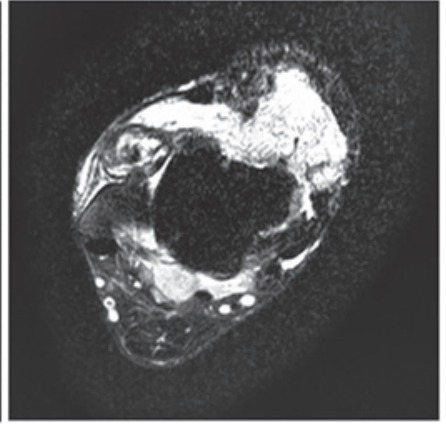

$\mathrm{T} 2 \mathrm{WI}$

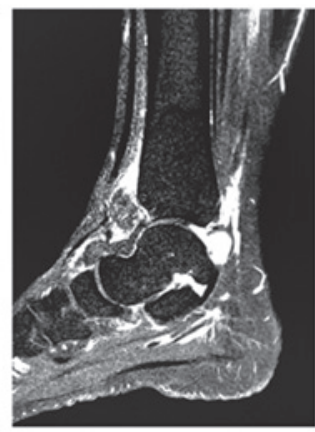

Gd-enhanced

Figure 3. Magnetic resonance image revealing a diffuse lesion that was isointense on T1WI and hypointense to hyperintense on T2WI (right, axial view on T1WI; middle, axial view on the T2WI; right, sagittal view on gadolinium-enhanced image). T1WI, T1-weighted image; T2WI, T2-weighted image; Gd, gadolinium.

sarcoma develops directly from synovial tissue is unclear (4). Approximately $5 \%$ of cases have been estimated to be intra-articular $(1,4)$. However, previous reports concerning synovial sarcoma suggest that intra-articular cases may be less common than originally thought $(1,6)$. Most cases of intra-articular synovial sarcoma originate in the knee joint. 
However, other locations have been reported, including one previously reported case involving intra-articular synovial sarcoma of the ankle (5), but no detailed clinical characteristics, such as tumor size, patient age, treatment method, and clinical course, were provided. Therefore, our case is the first reported case of intra-articular synovial sarcoma at the ankle whose clinical course has been described in detail. When intra-articular tumors are found, benign soft-tissue tumors, such as pigmented villonodular synovitis (PVNS) and synovial osteochondromatosis, are more frequently considered as differential diagnoses than synovial sarcoma. In our case, calcification was observed during a CT scan. Calcification is an extremely rare phenomenon in PVNS $(3,7,8)$, whereas $\sim 30 \%$ of cases involving synovial sarcoma exhibit calcification (9). On MRI, differentiating intra-articular synovial sarcoma from benign tumors is difficult and no certain radiological features have been established. Nordemar et al (3) reported that moderate and large amounts of joint effusion were only found in PVNS when they compared PNVS with synovial sarcoma. In the present case, the volume of joint effusion was low. However, other findings, such as extra-articular growth, low signal intensity in the synovia, and synovitis, were not significant variables on predicting synovial sarcoma (3). Synovial osteochondromatosis is also a rare joint disorder that is more rarely observed in the foot and ankle $(10,11)$. It is usually monoarticular and occurs most commonly in larger joints, with $>50 \%$ of the cases occurring in the knee $(10,11)$. Therefore, biopsy is often necessary to diagnose the patient, as described in the present case. We initially performed belowknee amputation, given the diffuse spread of the sarcoma throughout the ankle joint, which indicated that the foot could not be salvaged. We found five cases of intra-articular ankle soft tissue sarcomas in the literature $(12,13)$, including one case of extraskeletal myxoid chondrosarcoma and four cases of synovial chondrosarcomas. Amputation was performed in all five cases. Although limb salvage tumor resection was performed in patients with intra-articular soft tissue sarcoma at the knee joint (1-3), amputation may be necessary to resect the tumor completely due to widely diffused spread of the tumor. In conclusion, we experienced a case of intra-articular synovial sarcoma at the ankle joint. Although intra-articular primary soft tissue sarcomas are extremely rare, the possibility of malignancy should be considered. Moreover, when the tumor is widely spread, amputation may be performed to establish a clear surgical margin.

\section{Acknowledgements}

Not applicable.

\section{Funding}

No funding was received.

\section{Availability of data and materials}

All data generated or analyzed during this study are included in this published article.

\section{Authors' contributions}

TN conceived the study, treated the patients, collected the data and wrote the manuscript. TH collected, analyzed and interpreted the clinical data. KA performed surgery, and analyzed and interpreted the clinical data. AS analyzed and interpreted the clinical data, and reviewed the manuscript.

\section{Ethics approval and consent to participate}

The requirement for institutional review board approval from our institute was waived owing to the anonymized and retrospective nature of this report; however, written informed consent was obtained from the patient to perform further studies.

\section{Patient consent for publication}

Written informed consent was obtained from the patient to perform further studies.

\section{Competing interests}

The authors declare that they have no competing interests.

\section{References}

1. Friedman MV, Kyriakos M, Matava MJ, McDonald DJ, Jennings JW and Wessell DE: Intra-articular synovial sarcoma. Skeletal Radiol 42: 859-867, 2013.

2. Chebib I, Rosenberg AE, Fletcher CDM, Rosenthal DI, Hornicek FJ and Nielsen GP: Primary intra-articular sarcoma: A clinicopathological study of 15 cases. Histopathology 69: 614-623, 2016

3. Nordemar D, Öberg J, Brosjö O and Skorpil M: Intra-articular synovial sarcomas: Incident and differentiating features from localized pigmented villonodular synovitis. Sarcoma 2015: 903873, 2015.

4. Weiss SW and Goldblum JR (eds). Enzinger and Weiss's soft tissue tumors. 5th edition. Elsevier, St Louis, pp1161-1182, 2008.

5. Morton MJ, Berquist TH, McLeod RA, Unni KK and Sim FH: MR imaging of synovial sarcoma. AJR Am J Roentgenol 156: 337-340, 1991.

6. Cadman NL, Soule EH and Kelly PJ: Synovial sarcoma: An analysis of 134 tumors. Cancer 18: 613-627, 1965.

7. Murphry MD, Rhee JH, Fanburg-Smith LJC, Flemming DJ and Walker EA: From the archives of the AFIP pigmented villonodular synovitis: Radiologic-pathologic correlation. Radiographics 28: 1493-1518, 2009.

8. Oda Y, Izumi T, Harimaya K, Segawa Y, Ishihara S, Komune S, Iwamoto Y and Tsuneyoshi M: Pigmented villonodular synovitis with chondroid metaplasia, resembling chondroblastoma of the bone: A report of three cases. Mod Pathol 20: 545-551, 2007.

9. Bui-Mansfield LT and O'Brien SD: Magnetic resonance appearance of intra-articular synovial sarcoma: Case reports and review of the literature. J Comput Assist Tomogr 32: 640-644, 2008.

10. Saxena A and St Louis M: Synovial chondromatosis of the ankle: Report of two cases with 23 and 126 loose bodies. J Foot Ankle Surg 56: 182-186, 2017.

11. Derek Stensby J, Fox MG, Kwon MS, Cavcedo FJ and Rahimi A: Primary synovial chondromatosis of subtalar joint: Case report and review of the literature. Skeletal Radiol 47: 391-396, 2017.

12. Bhamra JS, Alorjani M, Skinner JA and Saifuddin A: Intra-articular extraskeletal myxoid chondrosarcoma of the ankle. Skeletal Radiol 41: 1017-1020, 2012.

13. Biazzo A and Confalonieri N: Synovial chondrosarcoma. Ann Transl Med 4: 280, 2016. 